\title{
Nucleos(t)ide analogue therapy for HBV-related HCC after hepatic resection: clinical benefits and unanswered questions
}

\author{
Jian-Hong Zhong
}

Received: 5 November 2014 / Accepted: 19 November 2014 / Published online: 28 November 2014

(C) International Society of Oncology and BioMarkers (ISOBM) 2014

Hepatic resection (HR) and radiofrequency ablation (RFA) remain the most frequent curative therapies for hepatocellular carcinoma (HCC) because of strict indications for liver transplantation and shortages of donated livers. Nevertheless, recurrence occurs in up to $75 \%$ of patients with intermediate or advanced HCC at 5 years after HR [1]; in these patients, 5year recurrence-free survival (RFS) is lower than $25 \%$, and tumor recurrence is the main cause of death [2]. Therefore, inhibiting tumor recurrence is a key to improving HCC patients' overall survival (OS).

Hepatitis B virus (HBV) is the most common cause of HCC around the world, which inspired clinicians to treat patients with HBV-related HCC using nucleos(t)ide analogues (NAs) to inhibit HBV replication and therefore reduce risk of HCC incidence. High HBV load and replication are associated with greater risk of HCC incidence [3], and large studies with long follow-up have shown that NA therapy can dramatically reduce HCC incidence and death in patients with liver cirrhosis who are chronically infected with HBV [4, 5]. NA therapy also appears to reduce risk of HCC recurrence after HR [6, 7], suggesting that it may improve OS for patients with HBVrelated HCC after curative HR or RFA.

Many retrospective studies have suggested that adjuvant NA therapy does reduce risk of HBV-related HCC recurrence after curative treatments [7-19]. Meta-analysis of these studies have concluded that NA therapy is associated with significantly lower tumor recurrence and liver-related mortality and significantly higher OS in patients with HBV-related HCC than no adjuvant therapy after curative treatments $[20,21]$. Unfortunately, the retrospective design of these studies limits

J.-H. Zhong $(\bowtie)$

Department of Hepatobiliary Surgery, Affiliated Tumor Hospital of Guangxi Medical University, He Di Rd. \#71, Nanning 530021, People's Republic of China

e-mail: zhongjianhong66@163.com the strength of their evidence. The strongest evidence of whether NA therapy offers clinical benefits would come, in principle, from a randomized controlled trial (RCT). However, carrying out an RCT is ethically and logistically difficult because oral NA therapy has already proven effective at preventing disease progression in patients chronically infected with HBV and because such therapy is becoming more affordable and does not cause significant side effects. Therefore, few patients with HBV-related HCC would volunteer for an RCT.

Despite these obstacles, Yin et al. managed to publish in 2013 the first RCT examining the efficacy of adjuvant NA therapy in 180 patients newly diagnosed with HBV-related HCC after curative HR [22]. All patients had serum HBV DNA levels greater than $100 \mathrm{IU} / \mathrm{mL}$. After median follow-up of 39.9 months, per-protocol analysis revealed that patients receiving lamivudine (100 $\mathrm{mg} /$ day) showed significantly higher short- and long-term RFS and OS than patients receiving no adjuvant therapy. In 2014, Huang and coworkers [23] performed an RCT examining the efficacy of adjuvant adefovir therapy in 200 patients newly diagnosed with HBVrelated HCC after curative HR. In contrast to the earlier RCT, all patients in this study had serum HBV DNA levels greater than $2000 \mathrm{IU} / \mathrm{mL}$. After median follow-up of 60 months, intention-to-treat analysis revealed that patients receiving adefovir (10 mg/day) showed significantly higher RFS and OS than patients receiving no adjuvant. Multivariate analysis showed that adefovir therapy was an independent protective factor of late tumor recurrence, which occurs $>2$ years after curative treatment and involves de novo tumorigenesis, but not of early $\mathrm{HCC}$ recurrence, which occurs $<2$ years after treatment and involves intrahepatic metastasis.

This evidence base of primarily retrospective studies and two RCTs (Table 1) makes a reasonably strong case that NA therapy offers clinical benefits in patients with HBV-related HCC after curative treatments like HR. However, there are 


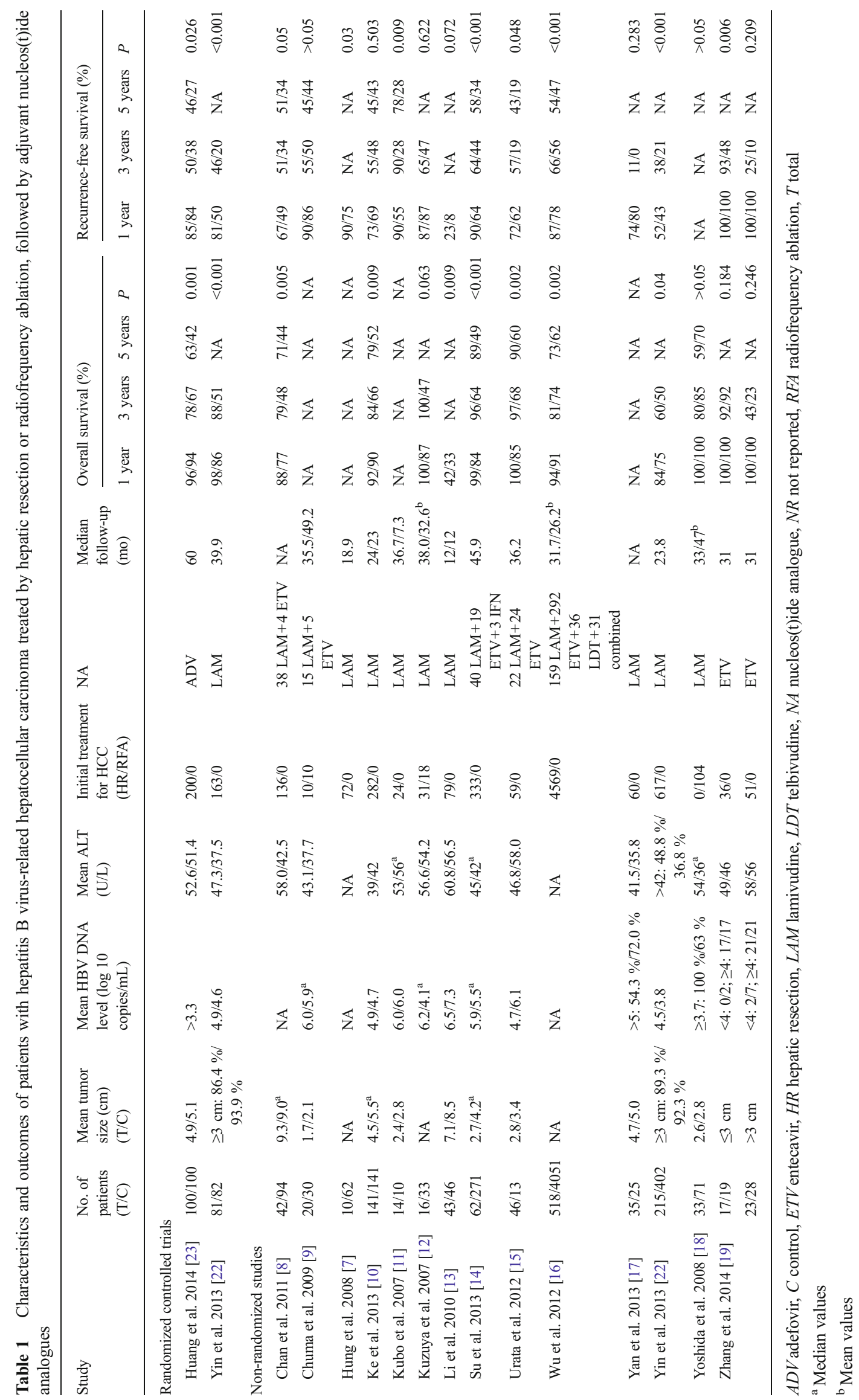


five major questions about NA therapy that have been neglected in the literature and that must be answered to maximize its efficacy in the clinic.

\section{How does NA therapy prevent HCC recurrence and improve OS?}

Though one RCT has reported that NAs significantly improve short- and long-term RFS [22], no study has directly established that the analogues exert antitumor effects. It is possible that NAs improve survival primarily by reducing HBV load. Lower HBV load in serum, indicative of reduced viral replication, is associated with lower risk of HCC occurrence in $\mathrm{HBV}$ carriers, irrespective of whether they have hepatitis [3, 4, 24]. Meta-analysis of eight studies involving 1610 patients with HCC chronically infected with HBV showed that lower viral load was associated with significantly lower risk of recurrence after HR or RFA [25]. Reducing HBV load improves genetic stability in hepatocytes and facilitates their regeneration following destruction by the virus.

The RCT by Huang et al. [23] reported that NA therapy reduced late $\mathrm{HCC}$ recurrence, but not early $\mathrm{HCC}$ recurrence. This is consistent with multivariate analysis of 193 patients with HBV-related HCC who underwent $\mathrm{HR}$, in which late recurrence was found to depend on high viral load and hepatic inflammation, whereas early recurrence depended on tumor factors [26].

Since NA therapy can reduce hepatitis activity and reduce chronic inflammation in the remnant liver after HR, it may improve survival by maintaining hepatic functional reserve. Therefore, the available evidence suggests that NA therapy improves OS in patients with $\mathrm{HBV}$-related $\mathrm{HCC}$ after $\mathrm{HR}$ through two main mechanisms: improving hepatic functional reserve and indirectly preventing late tumor recurrence. However, large, properly controlled studies are needed to test this hypothesis directly.

\section{When is the optimal time to initiate NA therapy, and how long should it last?}

Patients with HBV-related HCC suffer from both malignancy and chronic HBV infection, and although HR may treat the malignancy at least in the short term, it may also cause HBV reactivation. This can lead to fulminant hepatitis, liver failure, or even mortality. Post-resection rates of $\mathrm{HBV}$ reactivation range from 16 to $28 \%$ in patients not taking antiviral therapy [27-30], while the corresponding numbers are 0 and $2.9 \%$ in patients taking antiviral therapy [27-29]. Huang et al., the team that published one of the two RCTs on NA therapy [23], earlier investigated the risk of $\mathrm{HBV}$ reactivation in individuals with low serum levels of HBV DNA $(<2000$ IU/
$\mathrm{mL}$ ) at the time of $\mathrm{HR}$. [31] $\mathrm{HBV}$ reactivation occurred in $19.1 \%$ of patients by 1 year after surgery, and rates of liver failure were significantly higher in these patients than in those who did not suffer reactivation (10.5-11.8 \% vs. $2.7-6.4 \%$ ) [28, 31], while 3-year RFS and OS were significantly lower [31]. Given the strong potential for HBV reactivation and the fact that persistent HBV replication increases the risk of recurrence [32], we suggest that NA therapy be initiated before $\mathrm{HR}$ in patients with HBV-related HCC and detectable serum levels of HBV DNA. This recommendation should be examined directly in properly controlled trials.

Official guidelines for treating patients chronically infected with HBV differ in how long they recommend continuing NA therapy [33-35]. Moreover, it is unclear whether these guidelines are optimal or even appropriate for patients with $\mathrm{HBV}$ related HCC [36]. Since NA therapy cannot completely eradicate $\mathrm{HBV}$ and the treatment goal is to reduce HBV replication as much as possible to minimize the risk of reactivation, some investigators have advocated continuing antiviral treatment indefinitely, regardless of whether HBV DNA levels are undetectable or HBeAg seroconversion occurs [37, 38]. Those investigators argue that lifelong antiviral treatment may help prevent hepatitis flare-ups and maximally inhibit hepatocarcinogenesis.

Lack of official consensus on optimal NA therapy duration reflects a lack of studies on this question. In their RCT, Huang et al. [23] continued antiviral treatment unless there was unacceptable toxicity or withdrawal of consent. In contrast, Yin et al. kept the patients in their RCT on NA therapy until HBsAg seroconversion [22]. It is possible that halting NA therapy at HBsAg seroconversion may provide inadequate protection against future $\mathrm{HCC}$ recurrence or reactivation. Large, properly controlled trials are needed to examine the optimal duration of adjuvant NA treatment. These trials should take into account the problem of resistance to NAs that sometimes accompanies long-term NA monotherapy (see below).

\section{What are the indications for NA therapy?}

Whether NA therapy provides clinical benefit to all patients with HBV-related HCC undergoing HR is unknown. Current guidelines and clinical practices about patient selection for NA therapy revolve around three factors: serum level of HBV DNA, grade of liver function, and tumor stage. The literature provides no answers to the question of whether it is appropriate to administer adjuvant antiviral therapy to patients in whom serum levels of HBV DNA are undetectable or below the internationally recommended thresholds of $2000 \mathrm{IU} / \mathrm{mL}$. Many guidelines recommend such therapy only for patients with chronic HBV infection, evidence of active viral replication $(>2000 \mathrm{IU} / \mathrm{mL})$ and elevated levels of alanine 
aminotransferase. However, there is no international consensus on this point, in large part reflecting the fact that patients with low preoperative HBV load $(<2000 \mathrm{IU} / \mathrm{mL})$ can show HBV reactivation rates as high as $19.1 \%$ after HR. [31] The Chinese Medical Association [39] recommends that patients with HBV-related HCC take NAs as long as they show detectable levels of HBV DNA, regardless of alanine aminotransferase levels.

The question of whether and when to stop NA therapy is made even more complex by the fact that NAs can affect postHR outcomes related not only to HCC but also to remnant liver function. Cirrhosis in patients chronically infected with HBV strongly predicts HCC occurrence and disease-related mortality [40]. The finding that NAs can reduce hepatitis activity in such patients, coupled with their good tolerability, argues for prescribing analogues to most patients with HBVrelated $\mathrm{HCC}$ for as long as possible after HR, especially patients with progressive cirrhosis. Controlled trials are needed to address this question.

Since all patients in the two RCTs $[22,23]$ on NA therapy had Child-Pugh A liver function, and only some patients in the retrospective literature had Child-Pugh B liver function [10, 13], it is unclear whether patients with HBV-related HCC and Child-Pugh B or C liver function should receive adjuvant NA therapy after HR. The available evidence suggests that patients with reduced liver function can still benefit from this treatment. Lamivudine therapy rapidly suppressed HBV DNA load and improved the Child-Pugh score in patients with decompensated cirrhosis chronically infected with HBV [41, 42]. Antiviral therapy improved liver function in patients with HBV-related HCC [28, 43]. Large, controlled studies should examine directly whether NA therapy provides clinical benefits to patients with Child-Pugh B or C liver function.

The observation that NA therapy appears to affect primarily late recurrence, coupled with the high rate of early recurrence and low long-term OS of patients with advanced HCC, suggests that antiviral treatment should be more effective in patients with early-stage HCC. This hypothesis is supported by subgroup analysis of 478 patients with HBV-related HCC who underwent curative HR. [10] Adjuvant NA therapy was associated with significantly higher OS than no adjuvant antiviral therapy in HCC patients in Barcelona Clinical Liver Cancer (BCLC) stage A or B, but not in patients with BCLC stage $C$ disease [10]. Similarly, a retrospective study of 163 patients with HBV-related HCC found that NA therapy was associated with higher OS and RFS than no adjuvant antiviral therapy in patients with stage I or II tumors without major vascular invasion, but not in patients with stage III tumors or tumors of any stage showing major vascular invasion [8]. Finally, another retrospective study of 87 patients with HBVrelated HCC after curative HR found that NA therapy led to significantly higher RFS than no adjuvant antiviral therapy in patients with $\mathrm{HCC}$ tumors up to $3 \mathrm{~cm}$, but not in patients with tumors larger than $3 \mathrm{~cm}$ [19]. Given the relatively small populations in these studies, and their retrospective design, RCTs are needed to explore the efficacy of NA therapy in different HCC stages in the presence of different HCC comorbidities such as vascular invasion and portal hypertension.

\section{Which NA drug(s) should be used?}

Five NAs are currently used in the clinic to treat patients chronically infected with HBV: lamivudine, adefovir, telbivudine, entecavir, and tenofovir. While all these drugs are associated with similarly low toxicity, they can differ slightly in clinical efficacy and, most importantly, in how often they lead to resistance in patients on long-term monotherapy. Lamivudine monotherapy led to resistance in $22 \%$ patients in the RCT by Yin et al. [22] and to resistanceassociated breakthrough hepatitis in $14 \%$ of patients in a systematic review of four retrospective studies [44]. Adefovir monotherapy led to primary nonresponse in $3 \%$ of patients and resistance in $15 \%$ of patients in the RCT by Huang et al. [23] Telbivudine monotherapy was reported in one prospective study to lead to resistance in $10.8 \%$ of HBeAg-negative patients and $25.1 \%$ in $\mathrm{HBeAg}$-positive ones at 2 years [45]. Entecavir monotherapy for long-term treatment of chronic HBV infection led to resistance in $1.2 \%$ of patients in one prospective study [46]. Alone among the five NAs, tenofovir has been associated with a zero rate of resistance among patients with chronic hepatitis B [47]. Given the drug's antiviral potency and minimal toxicity, it may be the most suitable analogue. In fact, official guidelines [33-35] recommend entecavir and tenofovir as first-line antiviral therapy in patients chronically infected with HBV because of their superior ability to suppress viral replication and because of high genetic barriers to the development of resistance. However, we are unaware of RCTs or even retrospective studies comparing different NAs in parallel in patients with HBV-related HCC. Such studies should also specifically examine the perioperative use of entecavir and tenofovir.

Future studies should address the cost-effectiveness of NA therapy for HCC patients, since the literature has given conflicting results [48] and NA treatment costs remain prohibitive in HBV-endemic areas.

\section{Can multimodal treatment improve on NA therapy?}

Some evidence suggests that adjuvant or chemopreventive therapies may be useful supplements to NA therapy to further reduce late recurrence as well as inhibit early recurrence, thereby increasing survival from HCC $[49,50]$. These previous studies are retrospective and involve relatively small 
patient populations. Large RCTs should compare multimodal treatments with NA therapy alone.

In conclusion, the available evidence strongly suggests that adjuvant NA therapy in patients with HBV-related HCC improves liver function, significantly reduces late recurrence and improves OS following curative treatments such as HR. However, optimal implementation of NA therapy will require answering at least five unresolved questions: how the treatment prevents $\mathrm{HCC}$ recurrence and improves OS, when is the optimal time to initiate the treatment and how long it should be continued, for what patients the treatment is suitable, which NAs are the most cost-effective, and whether combining NA therapy with other treatments can further improve patient prognosis.

\section{References}

1. Zhong JH, Ke Y, Gong WF, et al. Hepatic resection associated with good survival for selected patients with intermediate and advancedstage hepatocellular carcinoma. Ann Surg. 2014;260(2):329-40.

2. Zhong JH, Wu FX, Li H. Hepatic resection associated with good survival for selected patients with multinodular hepatocellular carcinoma. Tumour Biol. 2014;35(9):8355-8.

3. Chen CJ, Yang HI, Su J, et al. Risk of hepatocellular carcinoma across a biological gradient of serum hepatitis B virus DNA level. JAMA. 2006;295(1):65-73.

4. Liaw YF, Sung JJ, Chow WC, et al. Lamivudine for patients with chronic hepatitis B and advanced liver disease. N Engl J Med. 2004;351(15):1521-31.

5. Wong GL, Chan HL, Mak CW, et al. Entecavir treatment reduces hepatic events and deaths in chronic hepatitis B patients with liver cirrhosis. Hepatology. 2013;58(5):1537-47.

6. Ko S, Nakajima Y, Kanehiro H, et al. Significant influence of accompanying chronic hepatitis status on recurrence of hepatocellular carcinoma after hepatectomy. Result of multivariate analysis. Ann Surg. 1996;224(5):591-5.

7. Hung IF, Poon RT, Lai CL, Fung J, Fan ST, Yuen MF. Recurrence of hepatitis B-related hepatocellular carcinoma is associated with high viral load at the time of resection. Am J Gastroenterol. 2008;103(7): 1663-73.

8. Chan AC, Chok KS, Yuen WK, et al. Impact of antiviral therapy on the survival of patients after major hepatectomy for hepatitis B virusrelated hepatocellular carcinoma. Arch Surg. 2011;146(6):675-81.

9. Chuma M, Hige S, Kamiyama T, et al. The influence of hepatitis B DNA level and antiviral therapy on recurrence after initial curative treatment in patients with hepatocellular carcinoma. J Gastroenterol. 2009;44(9):991-9.

10. Ke Y, Ma L, You XM, et al. Antiviral therapy for hepatitis B virusrelated hepatocellular carcinoma after radical hepatectomy. Cancer Biol Med. 2013;10(3):158-64.

11. Kubo S, Tanaka H, Takemura S, et al. Effects of lamivudine on outcome after liver resection for hepatocellular carcinoma in patients with active replication of hepatitis B virus. Hepatol Res. 2007;37(2): 94-100.

12. Kuzuya T, Katano Y, Kumada T, et al. Efficacy of antiviral therapy with lamivudine after initial treatment for hepatitis B virus-related hepatocellular carcinoma. J Gastroenterol Hepatol. 2007;22(11): 1929-35.
13. Li N, Lai EC, Shi J, et al. A comparative study of antiviral therapy after resection of hepatocellular carcinoma in the immune-active phase of hepatitis B virus infection. Ann Surg Oncol. 2010;17(1): 179-85.

14. Su CW, Chiou YW, Tsai YH, et al. The influence of hepatitis B viral load and pre-s deletion mutations on post-operative recurrence of hepatocellular carcinoma and the tertiary preventive effects by antiviral therapy. PLoS One. 2013;8(6):e66457.

15. Urata Y, Kubo S, Takemura S, et al. Effects of antiviral therapy on long-term outcome after liver resection for hepatitis B virus-related hepatocellular carcinoma. J Hepatobiliary Pancreat Sci. 2012;19(6): 685-96.

16. Wu CY, Chen YJ, Ho HJ, et al. Association between nucleoside analogues and risk of hepatitis B virus-related hepatocellular carcinoma recurrence following liver resection. JAMA. 2012;308(18): 1906-14.

17. Yan Q, Ni J, Zhang GL, et al. Efficacy of postoperative antiviral combined transcatheter arterial chemoembolization therapy in prevention of hepatitis B-related hepatocellular carcinoma recurrence. Chin Med J (Engl). 2013;126(5):855-9.

18. Yoshida H, Goto E, Sato T, et al. Safety and efficacy of lamivudine after radiofrequency ablation in patients with hepatitis $\mathrm{B}$ virus-related hepatocellular carcinoma. Hepatol Int. 2008;2(1):89-94.

19. Zhang ZY, Zhou ZQ, Zhou GW. Higher efficacy of antiviral therapy after major hepatectomy in patients with hepatitis B virus-related hepatocellular carcinoma of less than $3 \mathrm{~cm}$. Eur J Gastroenterol Hepatol. 2014;26(10):1116-24.

20. Sun P, Dong X, Cheng X, Hu Q, Zheng Q. Nucleot(s)ide analogues for hepatitis B virus-related hepatocellular carcinoma after curative treatment: a systematic review and meta-analysis. PLoS One. 2014;9(7):e102761.

21. Wong JS, Wong GL, Tsoi KK, et al. Meta-analysis: the efficacy of anti-viral therapy in prevention of recurrence after curative treatment of chronic hepatitis B-related hepatocellular carcinoma. Aliment Pharmacol Ther. 2011;33(10):1104-12.

22. Yin J, Li N, Han Y, et al. Effect of antiviral treatment with nucleotide/ nucleoside analogs on postoperative prognosis of hepatitis B virusrelated hepatocellular carcinoma: a two-stage longitudinal clinical study. J Clin Oncol. 2013;31(29):3647-55.

23. Huang G, Lau WY, Wang ZG, et al. Antiviral therapy improves postoperative survival in patients with hepatocellular carcinoma: a randomized controlled trial. Ann Surg. 2014.

24. Wong VW, Chan SL, Mo F, et al. Clinical scoring system to predict hepatocellular carcinoma in chronic hepatitis B carriers. J Clin Oncol. 2010;28(10):1660-5.

25. Qu LS, Liu JX, Kuai XL, Xu ZF, Jin F, Zhou GX. Significance of viral status on recurrence of hepatitis B-related hepatocellular carcinoma after curative therapy: a meta-analysis. Hepatol Res. 2014;44(7):750-60.

26. Wu JC, Huang YH, Chau GY, et al. Risk factors for early and late recurrence in hepatitis B-related hepatocellular carcinoma. J Hepatol. 2009;51(5):890-7.

27. Dan JQ, Zhang YJ, Huang JT, et al. Hepatitis B virus reactivation after radiofrequency ablation or hepatic resection for HBV-related small hepatocellular carcinoma: a retrospective study. Eur J Surg Oncol. 2013;39(8):865-72.

28. Lao XM, Luo G, Ye LT, et al. Effects of antiviral therapy on hepatitis $B$ virus reactivation and liver function after resection or chemoembolization for hepatocellular carcinoma. Liver Int. 2013;33(4):595-604.

29. Huang L, Li J, Lau WY, et al. Perioperative reactivation of hepatitis B virus replication in patients undergoing partial hepatectomy for hepatocellular carcinoma. J Gastroenterol Hepatol. 2012;27(1):158-64.

30. Kubo S, Nishiguchi S, Hamba H, et al. Reactivation of viral replication after liver resection in patients infected with hepatitis B virus. Ann Surg. 2001;233(1):139-45. 
31. Huang G, Lai EC, Lau WY, et al. Posthepatectomy HBV reactivation in hepatitis B-related hepatocellular carcinoma influences postoperative survival in patients with preoperative low HBV-DNA levels. Ann Surg. 2013;257(3):490-505.

32. Kim BK, Park JY, do Kim Y, et al. Persistent hepatitis B viral replication affects recurrence of hepatocellular carcinoma after curative resection. Liver Int. 2008;28(3):393-401.

33. Liaw YF, Kao JH, Piratvisuth T, et al. Asian-Pacific consensus statement on the management of chronic hepatitis B: a 2012 update. Hepatol Int. 2012;6:531-61.

34. Lok AS, McMahon BJ. Chronic hepatitis B: update 2009. Hepatology. 2009;50(3):661-2.

35. European Association for the Study of the Liver. EASL clinical practice guidelines: management of chronic hepatitis B virus infection. J Hepatol. 2012;57(1):167-85.

36. Ke Y, Wang L, Li LQ, Zhong JH. Nucleos(t)ide analogues to treat hepatitis B virus-related hepatocellular carcinoma after radical resection. World J Hepatol. 2014;6(9):652-9.

37. Wei $\mathrm{Q}, \mathrm{Xu} \mathrm{X}$, Ling $\mathrm{Q}$, Zheng S. Indefinite antiviral therapy may be required after surgical resection for hepatocellular carcinoma complicating chronic hepatitis B. J Res Med Sci. 2013;18(8):726-30.

38. Wei Q, Xu X, Ling Q, Zhou B, Zheng SS. Perioperative antiviral therapy for chronic hepatitis B-related hepatocellular carcinoma. Hepatobiliary Pancreat Dis Int. 2013;12(3):251-5.

39. Recommendation on antiviral therapy to hepatitis $\mathrm{B} / \mathrm{C}$ virus related hepatocellular carcinoma. Zhonghua Gan Zang Bing Za Zhi. 2013;21(2):96-100.

40. Ieluzzi D, Covolo L, Donato F, Fattovich G. Progression to cirrhosis, hepatocellular carcinoma and liver-related mortality in chronic hepatitis B patients in Italy. Dig Liver Dis. 2014;46(5):427-32.
41. Chu CM, Liaw YF. Hepatitis B virus-related cirrhosis: natural history and treatment. Semin Liver Dis. 2006;26(2):142-52.

42. Yao FY, Terrault NA, Freise C, Maslow L, Bass NM. Lamivudine treatment is beneficial in patients with severely decompensated cirrhosis and actively replicating hepatitis B infection awaiting liver transplantation: a comparative study using a matched, untreated cohort. Hepatology. 2001;34(2):411-6.

43. Kim JH, Park JW, Koh DW, Lee WJ, Kim CM. Efficacy of lamivudine on hepatitis B viral status and liver function in patients with hepatitis B virus-related hepatocellular carcinoma. Liver Int. 2009;29(2):203-7.

44. Zhong JH, Li le Q, Wu LC. Lamivudine with or without adefovir dipivoxil for postoperative hepatocellular carcinoma. Cochrane Database Syst Rev. 2011;(12):CD008713.

45. Liaw YF, Gane E, Leung N, et al. 2-Year GLOBE trial results: telbivudine is superior to lamivudine in patients with chronic hepatitis B. Gastroenterology. 2009;136(2):486-95.

46. Tenney DJ, Rose RE, Baldick CJ, et al. Long-term monitoring shows hepatitis $\mathrm{B}$ virus resistance to entecavir in nucleoside-naive patients is rare through 5 years of therapy. Hepatology. 2009;49(5):1503-14.

47. Snow-Lampart A, Chappell B, Curtis M, et al. No resistance to tenofovir disoproxil fumarate detected after up to 144 weeks of therapy in patients monoinfected with chronic hepatitis B virus. Hepatology. 2011;53(3):763-73.

48. Dusheiko GM. Cost-effectiveness of oral treatments for chronic hepatitis B. J Hepatol. 2009;51(4):623-5.

49. Zhong JH, Ma L, Li LQ. Postoperative therapy options for hepatocellular carcinoma. Scand J Gastroenterol. 2014;49(6):649-61.

50. Zhong JH, Zhong QL, Li LQ, Li H. Adjuvant and chemopreventive therapies for resectable hepatocellular carcinoma: a literature review. Tumour Biol. 2014;35(10):9459-68. 